\title{
A CONFIABILIDADE DO TRANSPORTE PÚBLICO POR ÔNIBUS EM JOÃO PESSOA (PB)
}

\author{
Paulo Vitor Nascimento de Freitas ${ }^{1}$ \\ José Augusto Ribeiro da Silveira ${ }^{2}$ \\ Alexandre Augusto B. da Cunha Castro ${ }^{3}$ \\ Ricardo Almeida de Melo ${ }^{4}$
}

\section{RESUMO}

Este trabalho objetiva mensurar a confiabilidade do sistema de transporte público urbano por ônibus de João Pessoa (PB), cidade média da região Nordeste do Brasil. Como método utilizou-se a análise estatística e geoespacial. Obtém-se a confiabilidade do tempo de viagem e o índice de cumprimento das viagens programadas. Os dados operacionais foram obtidos na Superintendência Executiva de Mobilidade Urbana de João Pessoa. Para tratamento estatístico dos dados e cálculo dos indicadores utilizou-se o software Microsoft Office Excel 2013. Os mapas temáticos foram construídos por intermédio do uso do software QGIS 8.8.2 Wien. Os resultados mostram que a confiabilidade do sistema é maior na área urbana mais consolidada, e deixa a desejar nos bairros mais distantes do centro localizados ao sul do município. Conclui-se que os indicadores e os produtos cartográficos gerados neste trabalho mostraram-se eficientes, possibilitando a identificação das linhas de ônibus e áreas da cidade que sofrem com problemas de confiabilidade do transporte público.

PALAVRAS-CHAVE: confiabilidade, transporte público, João Pessoa.

\section{RELIABILITY OF THE PUBLIC TRANSPORT BY BUS IN JOÃO PESSOA (PB)}

\section{ABSTRACT}

This study aims to measure the reliability of the urban public transport system by bus of the João Pessoa (PB), medium town in the Northeast of Brazil. As a method used the statistical and geospatial analysis. Obtains the reliability of travel time and the fulfillment index of the scheduled trips.

\footnotetext{
${ }_{1}^{1}$ Geógrafo e Mestrando em Engenharia Civil e Ambiental, PPGECAM/UFPB. paulogeo5@gmail.com.

2 Arquiteto e Urbanista e Professor do Programa de Pós-Graduação em Engenharia Civil e Ambiental, PPGECAM/UFPB. ct.laurbe@gmail.com.

3 Arquiteto e Urbanista, Mestre em Engenharia Urbana e Ambiental, UFPB. alexbccastro@hotmail.com.

${ }^{4}$ Engenheiro Civil e Professor no Programa de Pós-Graduação em Engenharia Civil e Ambiental, PPGECAM/UFPB. ricardo@ct.ufpb.br.
} 


\section{Revista Nacional de}

Gerenciamento de Cidades

definição dos planos de ação para melhoria do sistema, na medida em que dão suporte à identificação de suas partes deficientes. Para isso, tanto a parte técnicaoperacional como a opinião dos usuários precisam ser estudadas.

Dentre os atributos de qualidade do transporte público, a confiabilidade tem sido largamente considerada (Polus, 1978; Ferraz e Torres, 2004; Liu e Sinha, 2007; Silva, 2010). Ligado à consistência do serviço, este indicador é muito sensível à influência de fatores endógenos e exógenos, além de ser muito perceptível sob o ponto de vista do usuário.

Em João Pessoa, cidade média da região nordeste do Brasil que após a década de 1970 teve um rápido crescimento populacional, que promoveu uma expansão urbana acelerada principalmente na região sul do município, a elevação dos custos de urbanização tem dificultado a efetiva ampliação das redes de infraestrutura, inclusive aquelas voltadas para o sistema de transporte público (Oliveira, 2006). Sobre este sistema, Freire (2008) destaca que ele apresentava sinais de ineficiência e degradação.

Diante da importância da temática, este artigo objetiva mensurar a confiabilidade do sistema de transporte público urbano por ônibus de João Pessoa (PB). Trata-se de parte de uma pesquisa maior que versa sobre a qualidade do transporte público por ônibus de João Pessoa na visão dos usuários e de acordo com o desempenho técnico.

\section{O SISTEMA DE TRANSPORTE PÚBLICO POR ÔNIBUS DE JOÃO PESSOA}

O sistema de transporte público por ônibus da cidade de João Pessoa (figura 1) é operado por seis empresas, por meio do regime de concessão; são elas: Transnacional, Mandacaruense, Marcos da Silva, Reunidas, São Jorge e Santa Maria. A frota total do sistema é de 574 ônibus, cuja idade média é de 4,77 anos. Desta frota, 358 ônibus são adaptados para pessoas com mobilidade reduzida, chamados de "ônibus eficientes". O sistema dispõe de 86 linhas (sendo que duas 


\section{Revista Nacional de}

Gerenciamento de Cidades

desta cobertura no território da cidade revelou que os bairros centrais apresentaram um nível de acessibilidade elevado, enquanto que as áreas ao sul da cidade mostraram níveis menores. Este padrão também foi observado no estudo da frequência de atendimento das linhas de ônibus, que registrou os piores indicadores nas áreas mais periféricas da cidade (Freitas et al., 2015).

\section{CONFIABILIDADE EM SISTEMAS DE TRANSPORTE PÚBLICO}

A confiabilidade é um indicador do transporte público muito perceptível ao usuário. Sistemas pouco confiáveis não desempenham bem a função de atrair os usuários de modos individuais motorizados de transporte, pois significam muito tempo de espera nas paradas e atraso na chegada aos destinos, mesmo que o passageiro conheça a grade de horários.

Por isso, manter o sistema de transporte público com índices altos de confiabilidade é algo que traz benefícios ao operador e aos usuários (Polus, 1978). Serviços de alta confiabilidade atraem os usuários e tendem a diminuir os custos de operação.

Para Ferraz e Torres (2004), a confiabilidade do transporte público está relacionada ao grau de certeza dos usuários de que o veículo irá passar na origem e chegar ao destino dentro do horário previsto, considerando alguma margem de tolerância. Assim definida, pode-se dizer que a confiabilidade engloba tanto a pontualidade (grau de cumprimento dos horários) como também a efetividade na realização da programação operacional (porcentagem de viagens programadas realizadas). Polus (1978), por sua vez, define confiabilidade em transporte por ônibus como a capacidade de fornecer um serviço consistente ao longo de um determinado período de tempo.

A confiabilidade é um indicador do transporte público muito sensível e vulnerável a diversos fatores. Ela pode ser afetada por variáveis como características da via, falta de pessoal e controle do serviço, além das condições de trânsito - congestionamentos, por exemplo, impactam negativamente na 


\section{Revista Nacional de}

Gerenciamento de Cidades

Silva (2010) e Liu e Sinha (2007), por sua vez, calculam a confiabilidade por meio do inverso do coeficiente de variação, o que torna a variabilidade relativa à média de cada trecho estudado e também, assim como o inverso do desvio-padrão, manifesta uma correlação positiva entre o resultado e a qualidade do serviço quanto ao atributo.

Em geral, obtidos pelo inverso do desvio-padrão, as linhas com menores tempos de viagem tenderão a apresentar indicadores elevados. É razoável considerar que, para o usuário, um atraso de 5 minutos numa linha que leva em média 30 minutos para completar uma viagem não tem o mesmo peso que o mesmo atraso numa linha com tempo médio de viagem de 100 minutos, por exemplo. $\mathrm{Na}$ prática, pode-se dizer que variabilidades são mais perceptíveis e tendem a trazer mais insatisfação tanto quanto menor for o tempo de viagem. Por isso, considera-se que a mensuração da confiabilidade do tempo de viagem dado pelo inverso do coeficiente de variação, por tornar os dados relativos à média, apresentam indicadores com maior grau de correspondência à realidade. Por isso, neste trabalho, a confiabilidade do tempo de viagem é mensurada desta maneira.

Os indicadores são obtidos para cada linha de ônibus. Propõe-se que as viagens cujo tempo de duração são menores que a quarta parte da média ou maiores que três vezes a média sejam excluídas do cálculo do CTV e passem a ser consideradas como viagens não realizadas (que devem compor o cálculo do ICV), por dois motivos: podem se tratar de erros de entrada, uma vez que os registros de início e término da viagem são feitos manualmente; ou, mesmo sem erros de entrada, considera-se que variabilidades (neste caso, apenas os atrasos) fora deste intervalo rompem qualquer limiar aceitável de confiabilidade - na prática, é razoável supor que nestes casos a demanda do usuário por deslocamento não foi plenamente atendida. Após a exclusão das viagens que atendem a esta condição deve-se recalcular a média e o desvio-padrão, de modo a considerar apenas as viagens restantes. 


\section{Revista Nacional de}

Gerenciamento de Cidades

As expressões 1 e 2 foram utilizadas para obter a confiabilidade do tempo de viagem (CTV).

$$
\begin{gathered}
s=\sqrt{\frac{\sum_{i=1}^{n}\left(T r_{i}-T m\right)^{2}}{n-1}} \\
C T V=\frac{T m}{s}
\end{gathered}
$$

Onde: $\operatorname{Tr}:$ tempo de viagem realizado

Tm: tempo médio de viagem

$n: \quad$ número de viagens realizadas

\section{2 Índice de Cumprimento das Viagens programadas}

Um dos indicadores de qualidade do transporte coletivo mais utilizado pelos órgãos gestores, o Índice de Cumprimento das Viagens programadas (ICV) é dado pela razão do número de viagens realizadas e do número de viagens programadas (expressão 3).

$$
I C V=100 \cdot \frac{V R}{V P}
$$

Onde: VR: número de viagens realizadas

VP: número de viagens programadas

O ICV é calculado para cada linha de ônibus e dado em porcentagem. Quanto maior o valor, melhor é o indicador. $\mathrm{O}$ indicador do sistema é dado pela média dos indicadores das linhas. As viagens excluídas do CTV devem compor este índice. 

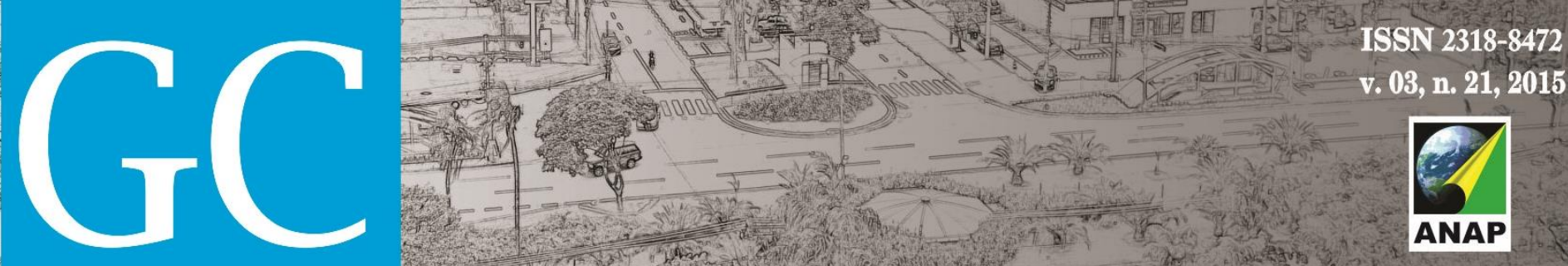

Revista Nacional de

Gerenciamento de Cidades

\section{RESULTADOS}

A tabela 1 mostra a posição que as linhas ocupam em relação ao sistema, segundo os indicadores, num universo composto por 84 linhas.

Tabela 1: Posição das linhas de ônibus em relação ao sistema segundo os indicadores obtidos

\begin{tabular}{|c|c|c|c|c|}
\hline \multirow{2}{*}{ Linha } & \multicolumn{2}{|c|}{ CTV } & \multicolumn{2}{|c|}{ ICV } \\
\hline & Posição & Indicador & Posição & Indicador \\
\hline 5605 & $2^{\circ}$ & 6,187503 & $1^{\circ}$ & 100 \\
\hline 3200 & $6^{0}$ & 5,524753 & $1^{0}$ & 100 \\
\hline 0304 & $1^{0}$ & 6,40121 & $12^{\circ}$ & 99,44444 \\
\hline 0303 & $13^{\circ}$ & 5,11718 & $1^{\circ}$ & 100 \\
\hline 3510 & $8^{\circ}$ & 5,421775 & $9^{\circ}$ & 99,6 \\
\hline A101 & $4^{0}$ & 5,683702 & $17^{\circ}$ & 99 \\
\hline 3507 & $22^{\circ}$ & 4,929929 & $1^{\circ}$ & 100 \\
\hline 5100 & $5^{0}$ & 5,631092 & $19^{\circ}$ & 98,82353 \\
\hline 5600 & $10^{\circ}$ & 5,246776 & $14^{\circ}$ & 99,375 \\
\hline 0107 & $26^{\circ}$ & 4,874786 & $1^{\circ}$ & 100 \\
\hline 0102 & $3^{\circ}$ & 6,151517 & $25^{\circ}$ & 98,46154 \\
\hline 5307 & $19^{\circ}$ & 4,966977 & $13^{\circ}$ & 99,44444 \\
\hline 2515 & $9^{\circ}$ & 5,254281 & $24^{\circ}$ & 98,5 \\
\hline 0204 & $27^{\circ}$ & 4,865926 & $10^{\circ}$ & 99,52381 \\
\hline 7118 & $17^{\circ}$ & 5,013858 & $20^{\circ}$ & 98,78788 \\
\hline 0201 & $29^{\circ}$ & 4,803961 & $11^{\circ}$ & 99,5 \\
\hline 0601 & $14^{\circ}$ & 5,11065 & $30^{\circ}$ & 97,25 \\
\hline 0600 & $18^{\circ}$ & 4,969655 & $28^{\circ}$ & 97,83784 \\
\hline 0203 & $30^{\circ}$ & 4,790919 & $18^{\circ}$ & 98,96104 \\
\hline 0513 & $33^{\circ}$ & 4,776163 & $15^{\circ}$ & 99,28571 \\
\hline 5206 & $11^{\circ}$ & 5,207677 & $45^{\circ}$ & 94,16667 \\
\hline 0604 & $20^{\circ}$ & 4,962659 & $37^{\circ}$ & 95,71429 \\
\hline 0202 & $12^{\circ}$ & 5,20302 & $46^{\circ}$ & 94,14634 \\
\hline 0510 & $16^{\circ}$ & 5,033323 & $43^{\circ}$ & 94,5679 \\
\hline 2514 & $28^{\circ}$ & 4,836013 & $31^{\circ}$ & 97,08333 \\
\hline 1500 & $44^{\circ}$ & 4,391875 & $16^{\circ}$ & 99,12088 \\
\hline 0116 & $32^{\circ}$ & 4,781111 & * & \\
\hline
\end{tabular}




\section{Revista Nacional de}

Gerenciamento de Cidades

Os produtos cartográficos gerados para a confiabilidade do tempo de viagem (figura 2) mostram que o extremo sudeste do município, onde opera a linha 1004, que faz o percurso Valentina/Praia do Sol, apresentou baixo indicador. Ao sudoeste, onde se localizam bairros como Bairro das Indústrias, Distrito Industrial e Mumbaba, os indicadores obtidos também foram baixos (linhas 1001, 103, 104 e 115, por exemplo). Ao leste do município, as linhas 507, 2307, 3207, 207 e 1007, responsáveis por atender os bairros Cabo Branco, Portal do Sol, Ponta do Seixas, Penha e Costa do Sol, também resultaram níveis baixos de confiabilidade do tempo de viagem.

Outra observação importante é a de que, na orla marítima, as linhas que atendem ao trecho mais ao norte (bairros do Bessa, Jardim Oceania e Aeroclube) apresentaram indicadores melhores do que o trecho mais ao sul. Ademais, as linhas do sistema com tempos de viagem mais confiáveis atendem a área urbana mais consolidada, enquanto que as áreas de expansão ao sul são atendidas por linhas que deixam a desejar com relação ao mesmo indicador.

No que diz respeito ao Índice de Cumprimento das Viagens (figura 3), a distribuição observada foi semelhante, embora parte das linhas que atendem aos bairros mais afastados e que apresentaram baixos indicadores de confiabilidade do tempo de viagem tenham apresentado indicadores melhores neste quesito, como é o caso das linhas 103, 104 e, principalmente, 1001. Para o sistema como um todo, o ICV obtido foi de $91,3 \%$. Apenas cerca de $10 \%$ das linhas cumpriram cem por cento das viagens programadas no período considerado.

As linhas transversais 5605 e 5600, que se caracterizam por não passarem pela área central e serem responsáveis, basicamente, por ligar dois importantes subcentros da cidade - os núcleos sudeste e litorâneo, conforme definidos por Andrade, Ribeiro e Silveira (2009) - e suas imediações, estão entre as linhas que apresentaram os melhores indicadores de CTV e ICV. A linha 5603, que completa o conjunto das linhas transversais e que também possui itinerário semelhante ao das outras duas, obteve indicador máximo para o ICV, mas um dos piores indicadores 


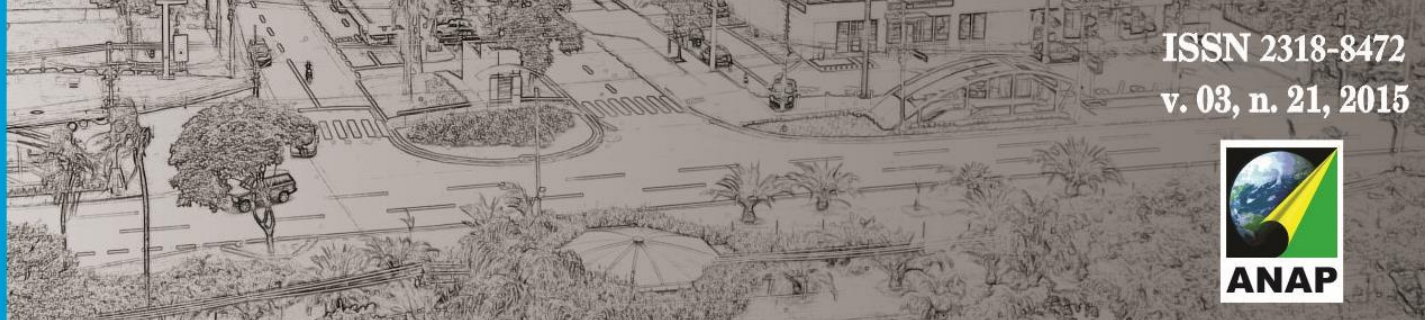

Revista Nacional de

Gerenciamento de Cidades

para o CTV. Observa-se que, de uma maneira geral, as linhas transversais obtiveram indicadores satisfatórios.

Percebe-se que, assim como estudos anteriores verificaram para a acessibilidade física aos pontos de ônibus e à frequência de atendimento das linhas (Freitas et al., 2015), a confiabilidade do sistema, tanto no que diz respeito ao tempo de viagem como também com relação ao cumprimento das viagens programadas, também deixa a desejar nos bairros mais distantes do centro localizados ao sul do município.

\section{CONSIDERAÇÕES FINAIS}

O transporte público é algo muito dinâmico e que pode sofrer a todo tempo alterações na programação operacional. Por isso, realizar estudos de confiabilidade para períodos muito grandes (meses, conjuntos de meses ou até estudos anuais) considerando o índice de cumprimento das viagens, por exemplo, embora seja o ideal, exige que o pesquisador tome conhecimento das alterações feitas na programação ao longo do recorte temporal do estudo para levá-las em consideração durante a fase de análise dos dados.

Por isso, como limitação, pode-se destacar o fato de que o período considerado pode não ser representativo para períodos maiores, como o de um ano, por exemplo. Como, no estudo aqui apresentado, não se dispôs das informações referentes às várias programações operacionais ao longo de períodos maiores, optou-se pelo período de uma semana típica recente.

Mesmo assim, os indicadores e os produtos cartográficos gerados neste trabalho mostraram-se eficientes. Foi possível, por meio de um estudo laboratorial, verificar onde circulam as linhas mais e menos confiáveis do sistema e, logo, quais áreas da cidade sofrem com problemas de confiabilidade do transporte público. Espera-se que os resultados desvencilhados e as análises efetuadas sirvam ao 
Revista Nacional de

Gerenciamento de Cidades

órgão gestor, de modo a direcionar atenção às linhas mal avaliadas visando à melhoria de seus indicadores de confiabilidade.

\section{AGRADECIMENTOS}

Ao Conselho Nacional de Desenvolvimento Científico e Tecnológico (CNPq), pelo auxílio financeiro dado por meio de bolsa de estudo concedida ao autor principal deste artigo; e à SEMOB-JP, pela solicitude na disponibilização dos dados necessários à realização do estudo.

\section{REFERÊNCIAS}

ANDRADE, P. A. F.; RIBEIRO, E. L.; SILVEIRA, J. A. R. Centralidade urbana na cidade de João Pessoa - PB: uma análise dos usos comerciais e de serviços entre o centro tradicional e o centro seletivo - 1970/2006. Arquitextos, São Paulo, 09.106. Vitruvius, mar 2009.

FERRAZ, Antonio Clóvis "Coca" Pinto; TORRES, Isaac Guillermo Espinosa. Transporte público urbano. São Paulo: Rima, 2004.

FREIRE, Paulo Sérgio Machado. O transporte urbano de João Pessoa. Minha Cidade, ano 08, 092 (04), 2008. Disponível em: [http://www.vitruvius.com.br/revistas/ read/minhacidade/08.092/1898]. Acesso em: 01/05/2014.

FREITAS, Paulo Vitor Nascimento de. SILVEIRA, José Augusto Ribeiro da; CASTRO, Alexandre Augusto B. da Cunha; SILVA, Lídia Pereira. Qualidade do transporte público urbano por ônibus: acessibilidade e frequência de atendimento em João Pessoa (PB). Anais do II ENURB, V SIMPGEU e II SINMA, Passo Fundo (RS), 2015. (No prelo)

LIU, Ronghui; SINHA, Shalini. Modelling urban bus service and passenger reliability. In: International Symposium on Transportation Network Reliability, Países Baixos, Jul, 2007. Disponível em: [http://www.its.leeds.ac.uk/software/dracula/downloads/Paper2-INSTR2007-Microsimulation-Bus-

Reliability-Liu.pdf] Acesso em: 30/09/2015.

OLIVEIRA, José Luciano Agra de. Uma contribuição aos estudos sobre a relação transportes e crescimento urbano: o caso de João Pessoa - PB. Dissertação de mestrado, Universidade Federal da Paraíba, 2006.

POLUS, Avishai. Modeling and measurements of bus service reliability. Transport Research. Vol. 12, 253-256, 1978.

SILVA, Thais Cristina Cunha e. A confiabilidade do transporte coletivo urbano em corredores estruturais de ônibus. Universidade Federal de Uberlândia, Programa de Pós-Graduação em Engenharia Civil, 2010. (Dissertação de mestrado)

SUPERINTENDÊNCIA EXECUTIVA DE MOBILIDADE URBANA DE JOÃO PESSOA - SEMOBJP. Dados operacionais sobre o transporte coletivo por ônibus de João Pessoa, 2015. (Resposta a requerimento de informação) 